\title{
Applications of Mixed Signal / SOC Technologies to Air Force Missions
}

\author{
Creigh Gordon \\ Electronics Engineer \\ Air Force Research Laboratory \\ (505) 846-6079 \\ creigh.gordon@kirtland.af.mil
}

\begin{abstract}
The Air Force Research Laboratory sees significant benefits in exploiting mixed technology system on chip in numerous areas of space mission data processing, and is moving towards developing technology for system integration on several fronts. Further technologies are being sought that could contribute to these objectives.
\end{abstract}

On board a satellite, data processing requirements form a pyramidal hierarchy. At the broad base of the pyramid, there is a range of simple control functions required in many places in a satellite. In the middle, there are more complex instrument control and some limited instrument data processing. At the top of the pyramid is the most complex mission data processing. Mixed technologies and systems on single chips can be applied on all levels, although the technologies tend to be applied differently at the various levels. At all levels, the benefits of mixed technology and system on a chip are considerable, and include lower cost, lower power, higher reliability, and increased capabilities. There are number of approaches and technology opportunities being investigated to realize these benefits and address the challenges.

Currently, the Air Force Research Laboratory is developing a single-chip microcontroller with numerous integrated functions useful for low- to medium-level instrument control. AFRL is also investigating the development of a single chip GPS receiver. At the highest levels, AFRL envisions high-performance processing nodes integrating embedded CPUs, RAMs, and reconfigurable FPGA blocks. The full paper will comment on possible future development strategies and consider how technology advances and limitations will affect on-board processing architectures. More than any other technology area, mixed signal and system-on-chip advancements could determine what new things future space systems are able to do, and how efficiently they do it.

\section{TABLE OF CONTENTS}

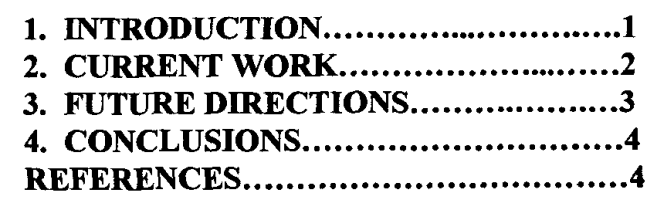

\section{INTRODUCTION}

Moore's Law tells us that transistor sizes are decreasing and counts are increasing. This has led to a revolution in data processing and computing, and certainly this revolution has been mirrored in the space computing world, albeit two or more generations behind the commercial market.

The holy grail of cell phone design is "antenna to bits" on a single chip. This requires the integration of various kinds of technologies (analog, digital, and memory) on one chip, which generally increases process complexity and may impose compromises in performance or increases in cost per chip. The benefits that flow from decreased part counts are well worth the effort, though; obviously lower system costs and greater reliability, and also reduced size and power consumption. These benefits are exactly the most critical to satellite system designers, and the Air Force Research Laboratory sees compelling reasons to pursue system-on-achip technologies that promise new capabilities and lower costs for space systems. Applications for mixed signal system-on-a-chip in information processing occur at various levels in a typical satellite. Some of the most interesting, near-term applications will be at a relatively low level. Here, at the embedded instrument or spacecraft microcontroller level, problems are tractably sized and solutions are widely usable. As technologies are developed for simple applications, they will naturally be then extended to higher-level, highly complex systems.

U.S. Government work not protected by U.S. copyright. The opinions expressed herein are those of the author, and should not be construed to represent official U. S. Govemment policy. 
Vol. 5-2412

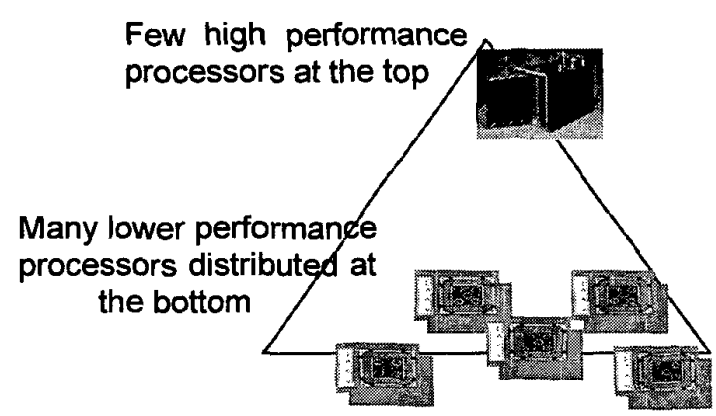

Fig.1 - Processing Hierarchy

The space computing world has more in common with embedded computing than it does with the more visible desktop computing environment. So, looking at trends in embedded computing, especially battery-powered, handheld systems, the Air Force sees increased integration not only (and maybe not even primarily) in more and more millions of transistors, but in increasing integration of system parts onto fewer and fewer semiconductor chips. In this conception, digital logic and memory are combined with analog processing on the same piece of silicon. The benefits for satellites from increasing this level of integration are smaller size and lighter weight, and perhaps even more importantly, lower power and higher reliability.

Although we eventually expect benefits of system-on-chip throughout the entire spectrum of spacecraft computing applications, initial projects are aimed at the lower end of the processing hierarchy. Here the principal functions are instrument control, and perhaps relatively simple instrument data processing. At this end of the pyramid, problems and projects are smaller and more tractable. And although it would seem that each application would be different enough to make it difficult for one system-on-chip architecture to cover a suitable range of uses, in fact it is not hard to provide enough computing horsepower to adapt one controller system to many uses.

There are significant problems in implementing systems on a single chip. But fortunately, it is quite possible to create partial solutions that are actually quite useful. For example, integrating memory and logic on a single chip is an increasingly interesting strategy. Adding nonvolatile storage is a dimension that many companies are investigating. There are rumors of nonvolatile elements on future Intel desktop chips. Such elements would provide a natural advantage for very low power, highly reliable embedded uses. Going further, adding analog elements such as A-to-D converters, filters, op amps, and other analog elements for true mixed signal processing is a goal.

Along the way, we'll have to solve both technical and economic problems. Design and test, yield and reliability, foundry access and low volume affordability are all potential issues. For the space market, radiation effects are an added complication owe have been able to find solutions for radiation effects in digital systems, but analog systems are likely to be more difficult.

Just as we expect to apply system-on-chip across the spectrum of applications, at AFRL we have attacked the problem on many levels. Some of our largest suppliers for space electronics have investigated mixed signal additions to their digital capabilities. And, in the past few years we have sponsored a number of SBIR topics that contributed towards system-on-chip capabilities. As noted before, partial solutions in this area can be quite useful, and even small companies with limited areas of expertise can contribute valuable technology.

\section{Current Work}

One of the Air Force Research Laboratory Space Vehicles Directorate's most advanced system-on-chip projects is a monolithic instrument controller. This controller is a highly integrated, self-supporting microcontroller node which can perform a variety of tasks of low to medium complexity. This single component contains an 8-bit microprocessor core (8051), EEPROM, SRAM, USARTs, ADC/DAC, and FIR filter elements. The controller will be packaged in a 68-pin quad flat pack for flight applications. This component is being designed by Mission Research Corporation using design-hardened (DH) circuit elements. Because of the DH technology, there are several foundry choices for fabrication that would yield satisfactory hardness for most space applications, while maintaining an affordable manufacturing cost structure. Fabrication at fully radiation hardened fab facilities is also a possiblility, for users who require higher radiation tolerance levels.

Another near-term AFRL project is the Miniaturized Mixed-Mode GPS Receiver (MMGR). A GPS receiver necessarily has significant $R F$ and logic functions, and when anti-jamming and anti-spoofing functions are added, there are considerable further complications. The MMGR project will develop technologies that are useful for these applications, with the ultimate goal of increased integration of GPS receivers intended for embedding down to the munitions level, as well as embedding in anything else one would want to locate.

The plan for MMGR is to implement building blocks for GPS designs, especially RF designs, into RF ASIC building 
blocks. These building blocks include low noise amplifiers, down converters, analog to digital converters, filter blocks, and analog adaptive processing blocks. Of course, these blocks would be useful for applications beyond GPS receivers, and could be combined on an RF ASIC for many purposes. Ultimately, they could be combined into a single RF ASIC, as a front end for a digital GPS receiver. While this project is not yet fully defined, it is very likely that the RF components will be developed on some kind of FET-oninsulator technology that will be compatible with complementary logic fabrication also. logic. But increasingly, design hardening is moving into analog and RF circuitry, and is being applied to mixed signal designs. Design hardening techniques might also be used to address a variety of problems besides radiation tolerance. For example, the hardened by design rules could be devised for decreased temperature sensitivity or increased reliability.

For the last two years, AFRL has also been pursuing research into nonvolatile memory technology based on

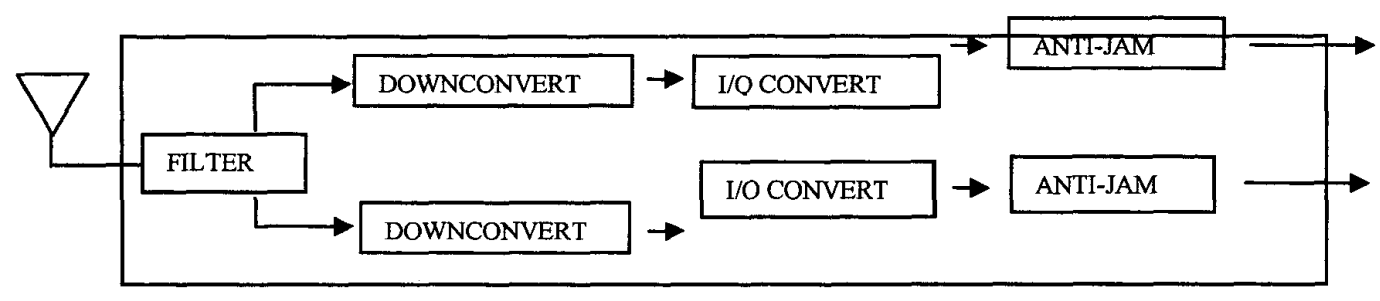

Fig. 2 - RF Blocks In A GPS Front End

An older project is the Mixed Signal Tile Array from Northrop Grumman. This project has designed an array of small-scale mixed signal circuits that can be configured for analog (and very limited digital) functions. Arrays can be tiled together to form more complex mixed signal components. Analog macro cells include voltage references and amplifiers, and discrete components include bipolar transistors and resistors. Digital macros include 70-gates of mask-programmed logic and 2 bits of nonvolatile memory. Each tile has $24 \mathrm{I} / \mathrm{O}$ pads. This product is fabricated on a 10-volt BiCMOS process, with 5-volt digital operation. The MSTA is intended to be widely applicable to analog and mixed signal problems because of its mix of components and interconnect flexibility. In general, digital processing has been adaptable and successful due to its high level of abstraction; MSTA is an attempt to increase the level of abstraction available for analog processing.

The Air Force Research Laboratory is also investing in design-hardening and fabrication techniques that may contribute to Mixed Signal/System-on-chip for future components. A major technology thrust for AFRL will be design and layout techniques that can be used at commercial foundries to manufacture radiation tolerant components for space use. These techniques include layout geometries that minimize the effects of trapped charges generated by ionizing radiation, and minimize sensitivity to single event effects. The techniques allow space electronics designers to leverage expensive advances in fabrication technology that will be made by commercial foundries. To date, design hardening techniques have been more focused on digital chalcogenide alloy. The particular chalcogenide compound has been used in CD-RW disks, which use a change in reflectivity between the crystalline and amorphous material phases. AFRL research uses a corresponding electrical resistivity change between phases to create a nonvolatile memory storage in a thin layer of alloy.

Because the level of crystallization (and thus the resistance) of the chalcogenide material can be controlled to any point between fully amorphous and fully crystallized by melting and controlled cooling, the memory cell is essentially a programmable resistor. This property could be exploited for a future multi-bit digital memory cell. The structure can also fairly easily be configured as a programmable resistor with size and speed commensurate with other integrated circuit structures. The programmable resistor characteristics are ideal for mixed signal and system-on-chip applications, and we believe that the potential exists for development of many innovative and yet-to-be imagined mixed signal devices.

At a more basic research level, AFRL is contributing to funding mixed signal research at several universities through the Center for Design of Analog-Digital Circuits (CDADIC) consortium. In these efforts, researchers from universities, government, and industry are designing mixed signal circuits, including phase lock loops and analog-todigital converters, and building test chips. The circuits are available for incorporation into products by the consortium members. 


\section{FUTURE DIRECTIONS}

In the future, mixed signal systems and system-on-chip developments will continue to play a large part in the AFRL space electronics development program. Certainly the chalcogenide-based technologies seem to hold the promise of many future applications. The digital world is continuously devising ways to incorporate analog functions with existing digital logic, and since AFRL has roots in the digital integrated circuit world, we have looked at ways to exploit this integration. There is also a certain amount of progress from the other side, where companies who have traditionally worked on linear and MMIC circuits are gradually adding logic gates to their analog circuitry. This reverse integration may also turn out to be a fruitful source of solutions for integrating analog and digital circuits.

In chalcogenide nonvolatile technologies, we have fairly specific roadmaps through 16 Mbit RAM devices, which are expected to be developed through 2004. Beyond that, chalcogenide developments are expected to focus on aspects other than straight RAM cell integration, perhaps into multibit storage, programmable logic device configuration storage, or other targets of opportunity.

We also expect to continue sponsoring small business innovative research (SBIR) topics that introduce one or more aspects of mixed signal electronics. These topics could be either explicitly mixed signal projects, or may incorporate mixed signal aspects simply as a competitive advantage in achieving a desired functional end result.

Design hardening projects, which are now mostly focused on digital technologies, will increasingly be targeted at analog functions, and will be targeted to foundries that have mixed signal capabilities. Design hardening techniques will have to be further developed for analog structures, a task that promises to be more subtle and complex than digital design hardening. This difficulty reflects the fact that analog technology is in many ways more subtle and complex than digital technology.

The big problem to overcome for analog systems is the one of generality. The digital electronic concept has been wildly successful in part because it reduces computing problems to very simple two-state expressions filtered through a small handful of elementary semiconductor structures. Analog, on the other hand, is much more difficult to generalize. Voltage levels and speeds vary, and it is impossible to define a complete set of elementary structures. As a result, analog frequently requires custom solutions; one size often doesn't very well fit all.

In the introductory paragraphs, it was noted that commercial applications such as cell phones are driving investments in these types of technologies. Since these applications are often very usable in satellite systems, AFRL will be following these developments and looking for ways to leverage them for space systems.

\section{Conclusions}

Increasing integration of analog and digital technologies is inevitable, although it may proceed more slowly than some might hope. This increasing integration is ideally suited for use in satellite electronics. The problem of hardening analog circuits is expected to be a difficult challenge. Yet partial success will result in useful, and each advance will act to spur further developments.

The Air Force expects mixed signal technologies to make tremendous advances in satellite capabilities in the near to mid-term future. Applications will be pervasive, in all the mission areas that the Department of Defense is interested in; surveillance, communications, and navigation. The Air Force Research Laboratory will most frequently attempt to adapt emerging commercial technologies (mixed signal design hardening). However there will be examples of space and military technology developments that are more advanced than commercial developments.

\section{REFERENCES}

1. ESA Seeks 'Smart' and Lighter Components. EE Times, Dec 5, 2002.

2. Leon Alkalai, "Advanced Microelectronics Technologies For Future Small Satellite Systems," Acta Astronautica V 46 N. 2-6 pp 233-239, 2000.

3. Glen H Fountain, et al., "A Technology Path To Distributed Remote Sensing," Acta Astronautica, v. 46 Nos 2-6 pp 251-261, 2000

4. Space Electronics Overview, MRC Microelectronics (www.mrcmicroe.com/spaceelect.pdf) July 2002.

5. Joop J.P Bruines, "Process Outlook for Analog and RF Applications," Microelectronic Engineering 54, pp. 35-48, 2000

Creigh Gordon is the Air Force technical program manager for numerous radiation hardened microelectronics developments, including the Advanced Spaceborne Computer Module (ASCM) program, Radiation Hardened FPGA, and Next Generation Space Processor (radiation hardened PowerPC 603e and 750) 